\section{Endoscopic trimming of a migrated gastroduodenal stent using a loop cutter and a two-channel endo- scope}

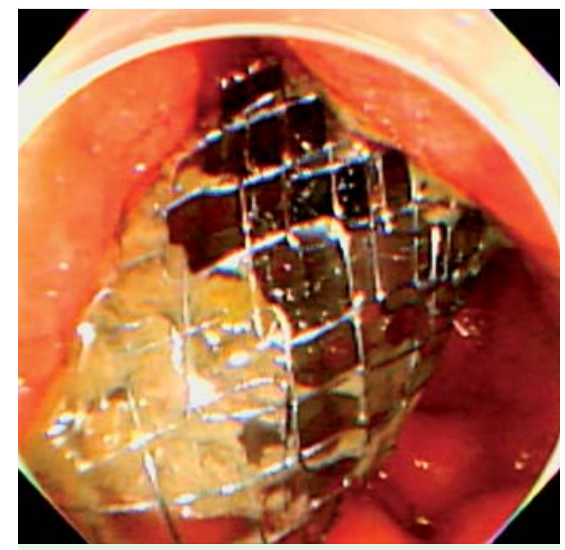

Fig. 1 Endoscopic view of migrated gastroduodenal stent.

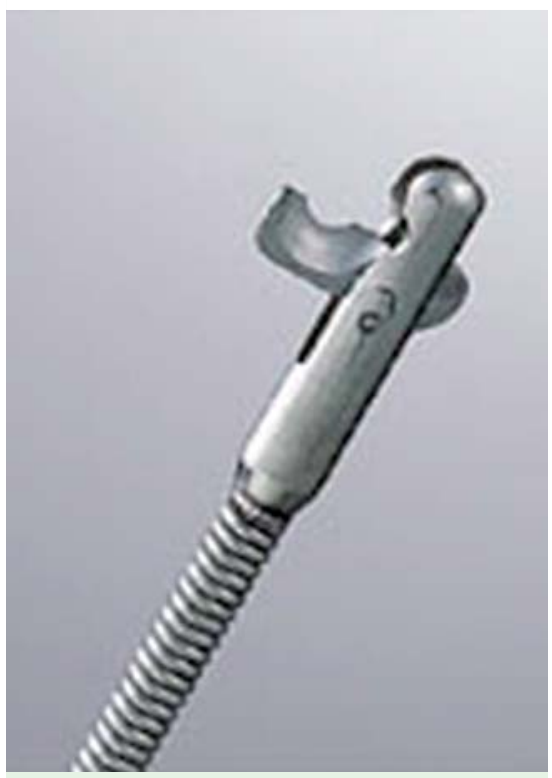

Fig. 2 Loop cutter FS-5L-1 device (Olympus Medical Systems Corp., Tokyo, Japan).

A 69-year-old man with gastric cancer was admitted to our hospital because of vomiting. Two gastroduodenal stents (Niti-S D pyloric/duodenal stent; TaeWoong Medical Inc., Seoul, South Korea) had previously been inserted from the first portion of the duodenum to the body of the stomach because of antral obstruction. Esophagogastroduodenoscopy revealed migration of the proximal stent ( $\bullet$ Fig. 1). The stent required trimming to resolve the symptoms.

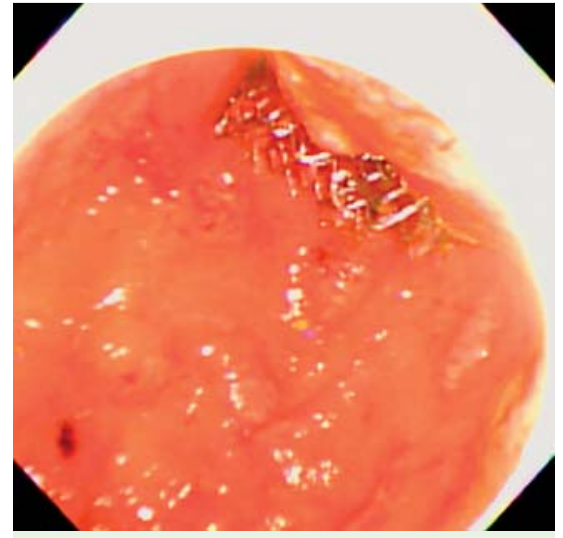

Fig. 3 Endoscopic view of gastroduodenal stent after trimming.

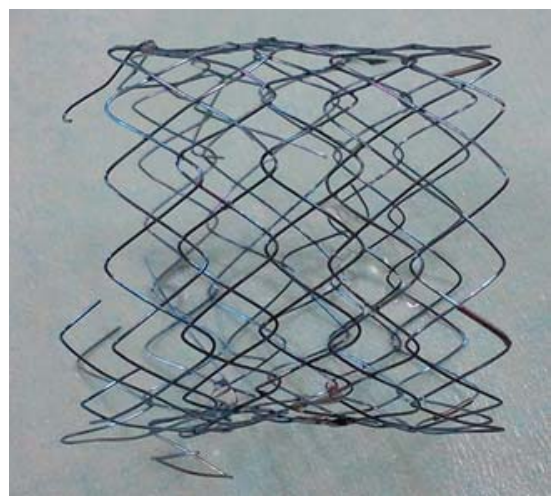

Fig.4 The trimmed gastroduodenal stent.

A loop cutter FS-5L-1 (Olympus Medical Systems Corp., Tokyo, Japan) was used to trim the stent ( $\bullet$ Fig. 2). The GIF-2T240 (Olympus) two-channel endoscope was used for the procedure. The wire was easily cut by the loop cutter device when a single wire was grasped precisely. If the wire became stuck in the groove of the scissors, another loop cutter was inserted through the opposite working channel in order to cut the stuck wire ( $\bullet$ Video $\mathbf{1}$ ). After the stent had been cut circumferentially, only the section that was embedded deeply into the tumor was left. The wires near the embedded site were grasped tightly and were torn away from the tumor. Finally, the trimmed stent was retrieved by alligator forceps through the overtube ( $\bullet$ Fig. 3 and $\odot$ Fig. 4 ).
There are several reports describing the trimming of biliary metal stents, but reports of enteral stent trimming are limited [1-3]. Argon plasma coagulation (APC) is frequently reported as a stent trimming device, whereas reports on the use of the loop cutter are limited [4,5]. The major problem associated with the use of APC is mucosal damage, especially when the stent wire is embedded into the tumor. The major problem with the loop cutter is that the wire sometimes becomes stuck in the groove of the scissors. To overcome this problem, a two-channel endoscope is used and another loop cutter is inserted to cut the stuck wire if needed. Therefore, trimming the Niti-S D pyloric/duodenal stent using a loop cutter and two-channel endoscope is safe and useful.

\section{Video 1}

A Niti-S D pyloric/duodenal stent (TaeWoong Medical Inc., Seoul, South Korea) was trimmed using a loop cutter. When the wire became stuck in the groove of the scissors, another loop cutter was inserted through the opposite working channel to cut the stuck wire.

Endoscopy_UCTN_Code_TTT_1AO_2AZ

Competing interests: None

Takashi Sasaki' , Hiroyuki Isayama', Shuntaro Yoshida ${ }^{1}$, Yousuke Nakai ${ }^{1}$, Hiroharu Yamashita², Yasuyuki Seto ${ }^{2}$, Kazuhiko Koike ${ }^{1}$

${ }^{1}$ Department of Gastroenterology, Graduate School of Medicine, University of Tokyo, Tokyo, Japan

2 Department of Gastrointestinal Surgery, University of Tokyo, Tokyo, Japan

\section{References}

1 Guda NM, Freeman ML. Endoscopic transection of distally migrated biliary self-expandable metallic stents by using argon plasma coagulation: a report of 2 cases (with video). Gastrointest Endosc 2006; 63: 12 - 14

2 Hamada T, Nakai $Y$, Isayama $H$ et al. Trimming a covered metal stent during hepaticogastrostomy by using argon plasma coagulation. Gastrointest Endosc 2013; 78: 817

3 Witte TN, Danovitch SH, Borum ML et al. Endoscopic trimming of a rectal self-expandable metallic stent by use of argon plasma coagulation. Gastrointest Endosc 2007; 66: $210-211$ 
4 Vanbiervliet G, Piche T, Caroli-Bosc FX et al. Endoscopic argon plasma trimming of biliary and gastrointestinal metallic stents. Endoscopy 2005; 37: 434-438

5 Maetani I, Shimura J, Ukita T et al. Successful repair of a damaged duodenal stent by cutting stent wires and placement of a second stent. Endoscopy 2002; 34: 86-88
Bibliography

Dol http://dx.doi.org/

10.1055/s-0034-1377552

Endoscopy 2014; 46: E462-E463

(c) Georg Thieme Verlag KG

Stuttgart · New York

ISSN 0013-726X

\section{Corresponding author}

Takashi Sasaki, MD, PhD

Department of Gastroenterology

Graduate School of Medicine

University of Tokyo

7-3-1 Hongo, Bunkyo-ku

Tokyo 113-8655

Japan

Fax: $+81-3-38140021$

sasakit-tky@umin.ac.jp 